ORIGINAL ARTICLE

\title{
Temporal association between serum prolactin concentration and exposure to styrene
}

\author{
U Luderer, R Tornero-Velez, T Shay, S Rappaport, N Heyer, D Echeverria
}

Occup Environ Med 2004;61:325-333. doi: 10.1136/oem.2002.005561

See end of article for authors' affiliations ....................

Correspondence to: Dr U Luderer, Center for Occupational and Environmental Health, 5201 California Avenue, Suite 100, Irvine, CA 92612, USA; uluderer@ uci.edu

Accepted

5 September 2003
Background: Previous studies have suggested that occupational exposure to styrene is associated with increased serum levels of the anterior pituitary hormone prolactin (PRL).

Aims: To test the hypotheses that: (1) the effect of styrene exposure on PRL secretion is an acute effect, not a subchronic or chronic effect; (2) blood styrene, as a measure of absorbed dose, is a stronger predictor of serum PRL level than personal breathing zone air styrene concentration.

Methods: Subjects were recruited from 17 workplaces in the reinforced plastics industry. Personal breathing zone air styrene, whole blood styrene, and serum PRL were measured during one to three sessions, approximately one year apart. Linear multiple regression was used to model the relations between acute (air styrene or blood styrene obtained at same time as PRL), subchronic (average air or blood styrene over two or three sessions), and chronic (years of work in industry or facility times average air styrene over all sessions) indices of styrene exposure and serum PRL.

Results: Acute blood styrene concentration was the strongest predictor of serum PRL concentration, with the model predicting a 2.06-fold increase in PRL $(95 \% \mathrm{Cl} 1.11$ to 3.84$)$ for every 10 -fold increase in blood styrene. Serum PRL tended to increase with increasing styrene exposure in both men and women; however, women tended to have higher PRL levels. For women, the change in blood styrene between sessions 1 and 2 was a significant predictor of the change in serum PRL between sessions.

Conclusions: Results confirm that styrene exposure enhances serum PRL concentrations and support an acute effect of styrene on PRL secretion.
$\mathrm{T}$ his study evaluates whether an association between increased serum levels of the hormone prolactin (PRL) and styrene exposure is fundamentally an acute response in contrast to potentially less pronounced associations with subchronic and chronic exposure to styrene. Styrene is a widely used aromatic chemical to which an estimated 330000 workers are exposed yearly in the United States. ${ }^{1}$ Occupational exposure limits for styrene have been set based primarily on neurotoxicity. At exposure levels below $100 \mathrm{ppm}$, some human studies have reported no significant effects on neurobehavioural test outcomes; ${ }^{23}$ whereas others observed reduced performance on some neurobehavioural measures. ${ }^{4-7}$ However, other endpoints indicative of neurotoxicity have been observed at lower exposure levels. Acquired dyschromatopsia has been consistently associated with styrene exposure levels less than 100 ppm. $^{8-10}$ Several studies have found increased serum PRL concentrations in both men and women exposed to mean styrene levels less than $100 \mathrm{ppm}$ compared to unexposed controls. ${ }^{11-13}$ The American Conference of Government Industrial Hygienists (ACGIH), as well as the governments of Germany, Finland, Spain, Italy, and Austria, recommend an eight hour time weighted average threshold limit value (TLV) of 20 ppm. ${ }^{14}{ }^{15}$ The United States Occupational Safety and Health Administration permissible exposure limit (OSHA PEL) is $50 \mathrm{ppm}$, as are the occupational exposure limits in France, Belgium, and Ireland. ${ }^{15}$

PRL is an anterior pituitary hormone that controls milk production. In addition, small amounts of PRL are synthesised in many other tissues, including brain, uterus, and immune cells. ${ }^{16}$ PRL secretion by the pituitary is under tonic inhibitory control by hypothalamic dopamine secreted by the tuberoinfundibular dopaminergic neurones. Studies in the rabbit ${ }^{17}$ and the rat ${ }^{18}$ have shown reduced tuberoinfundibular dopamine levels following styrene exposure. These findings suggest that the increased PRL levels observed in styrene exposed workers may be caused by styrene induced suppression of hypothalamic dopamine.

Previous studies used urinary concentrations of the styrene metabolites mandelic acid and phenylglyoxylic acid ${ }^{11-13}{ }^{19}$ and ambient air styrene concentrations ${ }^{12}{ }^{13}$ as markers of styrene absorbed dose or exposure, respectively. The metabolism of styrene, and thus the concentrations of its urinary metabolites, is altered by coexposures with other solvents, such as ethanol. ${ }^{20}$ Moreover, the urinary metabolites are also produced by the metabolism of solvents such as ethylbenzene and phenylglycol and of some drugs. ${ }^{21}$ Ambient air styrene concentrations may not reflect individual exposures, and they are markers of exposure rather than absorbed dose. Therefore, we sought to correlate serum PRL concentration with whole blood styrene levels. Previous studies were crosssectional in design, and, therefore, also did not examine the role of acute versus chronic styrene exposure on PRL levels. We therefore measured styrene and PRL repeatedly over time in the same individuals, and obtained detailed exposure histories in order to determine whether styrene exposure levels measured simultaneously with PRL were more strongly associated with serum PRL than subchronic (1-2 years) or chronic (many years) indices of styrene exposure.

This study was designed to test the hypotheses that: (1) the effect of styrene on PRL secretion is an acute effect, not a subchronic or chronic effect; and (2) blood styrene, as a measure of absorbed dose, is a stronger predictor of serum PRL level than personal breathing zone air styrene concentration.

Abbreviations: $A C G H$, American Conference of Government Industrial Hygienists; PEL, permissible exposure limit; PRL, prolactin; TLV, threshold limit value 
Main messages

- Serum prolactin concentrations increased with increasing blood styrene concentrations and with increasing personal breathing zone air styrene levels.

- Blood styrene concentration obtained at the same time as serum prolactin concentration was a better predictor of serum prolactin concentration than was full-shift time weighted average air styrene concentration or calculated indices of subchronic or chronic styrene exposure.

- Exposure to full-shift time weighted average air styrene levels greater than 20 ppm was associated with increased risk of increased serum prolactin concentrations above the normal reference range.

\section{METHODS}

\section{Experimental design}

The design was a cohort study in which employees at 17 workplaces in the reinforced plastics industry (four boat repairers, seven boat manufacturers, one bathtub manufacturer, two truck/recreational vehicle manufacturers, three pipe and tank manufacturers) were recruited for sequential measurements (two or three sessions approximately one year apart) of personal breathing zone styrene and acetone levels and blood sampling for styrene and PRL. At the time of sampling, interviews were also conducted, and the individuals were observed while performing their job tasks. This was a part of a larger study designed to evaluate the effects of styrene on neurological outcomes. Consistent with the Declaration of Helsinki, the study was approved by the Human Subjects Committees of the University of Washington, Battelle CPHRE, and the University of North Carolina School of Public Health, and informed consent explaining the purpose and procedures of the study was obtained from all study participants.

\section{Interviews and observation}

On the days of the blood draws, structured interviews were conducted by trained interviewers. Information gathered included demographic characteristics, employment history, medical history, height, weight, tobacco use history, alcohol use history, and dietary information. The interviewers also observed each individual during the course of their work shift and recorded information about job tasks performed, whether the individual was working in a confined space, and whether the individual was using a respirator, gloves, protective clothing, hearing protection, and safety glasses.

\section{Exposure assessment}

For each session, an average of 3 (range 1-5) personal breathing zone full-shift styrene measurements were made on separate workdays over a one month period. Blood was collected by venipuncture for PRL and styrene measurements within 10 minutes of the end of the shift on one of these days, which was also the day of the interview and observation. Aliquots of whole blood were immediately mixed with internal standards in a mobile laboratory on site and shipped at $-80^{\circ} \mathrm{C}$ to the University of North Carolina where styrene concentrations in whole blood were measured by isotope dilution gas chromatography-mass spectrometry (GC-MS). ${ }^{22}$ Briefly, a $2 \mathrm{ml}$ aliquot of whole blood was transferred to an $8 \mathrm{ml}$ vial containing $4 \mathrm{ml}$ of $n$-pentane and $2 \mu \mathrm{g}$ of $\left[{ }^{2} \mathrm{H}_{8}\right]$-styrene as internal standard. The vial was sealed with a Teflon lined screw cap, the contents were mixed

\section{Policy implications}

- Occupational exposure to styrene at air levels currently permissible in many countries is associated with increased serum prolactin levels.

- While the observed increases in serum prolactin are of uncertain clinical significance, the results suggest that further lowering of occupational exposure limits for styrene may be warranted.

with a vortex mixer for 30 seconds, and the phases were separated by centrifugation at $800 \mathrm{~g}$ for 5 minutes. The pentane extracts were stored in $8 \mathrm{ml}$ vials with Teflon lined screw caps at $-80^{\circ} \mathrm{C}$ for up to one month before analysis. The limit of detection of this assay was $0.001 \mathrm{mg} / \mathrm{l}$. Another aliquot of whole blood was processed by centrifugation, serum was collected, frozen at $-20^{\circ} \mathrm{C}$, and shipped to the University of Washington where PRL assays were performed.

Full work shift (approximately eight hours) personal styrene and acetone concentrations were measured with $3 \mathrm{M}$ Organic Vapor passive air sampling monitors. The monitors contained activated carbon as the adsorbent and relied on diffusion to transfer analyte from the air. After sampling, the monitors were capped and shipped at room temperature to the University of North Carolina, where they were stored for up to 24 hours, and desorbed with $1.5 \mathrm{ml}$ of ethlyacetate. The solution was decanted into a $4 \mathrm{ml}$ glass vial, sealed with a Teflon lined cap, and stored at $-20^{\circ} \mathrm{C}$ for up to one month prior to analysis. Analysis involved injection of $1 \mu \mathrm{l}$ of the solution into a GC system equipped with flame ionisation detection. Analytes were corrected for blanks (at least one unexposed monitor per five exposed) and for desorption efficiency. ${ }^{23}$ The limit of detection of this assay was 1 ppm.

For the purposes of the statistical analyses, levels of blood and air styrene below the limits of detection were arbitrarily set equal to two thirds of the limit of detection.

\section{Prolactin assay}

Serum PRL concentrations were measured at the University of Washington Clinical Research Laboratories using the Emulyte chemiluminescent immunoassay (DPC, Los Angeles, CA). The limit of detection of the assay was $1 \mathrm{ng} /$ $\mathrm{ml}$. For control samples with known concentrations of $8 \mathrm{ng} /$ $\mathrm{ml}, 15 \mathrm{ng} / \mathrm{ml}$, and $35 \mathrm{ng} / \mathrm{ml}$, the interassay coefficients of variation were $6 \%, 4 \%$, and $4 \%$, respectively. The 95 th centile for the laboratory reference value for PRL levels was $>15 \mathrm{ng} /$ $\mathrm{ml}$ for males and $>20 \mathrm{ng} / \mathrm{ml}$ for females.

\section{Statistical analysis}

Blood and air styrene concentrations as well as serum PRL concentration were transformed to logarithms (base 10) to satisfy normality and equal variance assumptions. Bivariate analyses were performed to assess the effects of various potential confounders on blood and air styrene and on serum PRL. The independent samples $t$ test (of logged observations) was used to test the effect of dichotomous variables (gender, smoking, alcohol use, use of styrene based food and beverage containers, use of a respirator, wearing gloves while using styrene, working in a confined space, use of recreational drugs, use of prescription drugs, use of over-the-counter drugs). One way analysis of variance was used to make multi-group comparisons (ethnicity, industry, number of cigarettes smoked per day, number of alcoholic beverages per week). The Pearson correlation was used to evaluate the relation of continuous potential confounding variables (air 
acetone concentration, age, body mass index, income) with exposure and PRL levels. The bivariate analyses were performed on the first session from each individual to avoid any effect of multiple sessions per person. Biological plausibility, previous research findings, and a p value $<0.20$ in the bivariate analyses were used as criteria to select the variables for the initial linear regression modelling.

PRL was modelled as a function of acute, subchronic, or chronic air or blood styrene using linear multiple regression. For the acute exposure model, air or blood styrene concentrations obtained on the same day as the PRL concentration were used. For the subchronic exposure models air or blood styrene values were averaged over all the study sessions, if sampling occurred during more than one session for that individual. For the chronic exposure models all the air styrene values for a given individual were averaged and multiplied by the number of years that individual had worked in the styrene industry or the number of years the individual had worked in the styrene facility where he or she was currently employed. To test whether current serum PRL concentration was associated with styrene exposure during the preceding one to two years (subchronic) or with exposure over multiple years (chronic), the outcome variable for the subchronic and chronic analyses was the serum PRL concentration for the last study session in which the individual participated (the second or third session). Because serum PRL levels are higher in women than in men, gender was forced into the models. Various models were tested with the potential confounding variables identified by the bivariate analyses, and only age and smoking were observed to be significant predictors of PRL concentration. Thus, the summary models presented included gender, smoking, and age in addition to the exposure variable. In addition, because repeated determinations of PRL or styrene concentrations on the same individual are not independent observations, data from session 1 and session 2 were analysed separately in acute models. Control for age was approached by directly including it in the models. However, we also controlled for age and other inter-individual differences by using each subject as his/her own control by predicting the difference between session 2 and session 1 serum PRL levels as a function of the difference between session 2 and session 1 blood styrene levels.

To assess whether styrene exposure at levels above the ACGIH TLV of 20 ppm was associated with an increased risk of having a clinically increased serum PRL concentration (greater than $15 \mathrm{ng} / \mathrm{ml}$ for men or $20 \mathrm{ng} / \mathrm{ml}$ for women), air styrene was also dichotomised into groups of less than or greater than the ACGIH recommended TLV of $20 \mathrm{ppm}$ $(\leqslant 20 \mathrm{ppm} v>20 \mathrm{ppm})$ and serum PRL was dichotomised into groups of less or greater than the upper limits of normal for PRL. The association between serum PRL and air styrene was then assessed by logistic regression analysis with adjustment for the same confounding variables as the linear regression analyses.

The SPSS statistical package (version 10.0 for the Macintosh) was used for all statistical analyses (SPSS, Chicago, IL).

\section{RESULTS}

\section{Characteristics of the study population}

Table 1 shows demographic characteristics of the study population. There were 259 men and 43 women in the study population. PRL concentrations and blood styrene measurements were available on 206 individuals ( 33 women) for 386 sessions. PRL and air styrene concentrations were available for 228 individuals ( 35 women) and 417 sessions, respectively. The mean ages of the study subjects were 35.6 years for the men and 37.7 years for the women. Sixty eight per cent of the workers were non-Hispanic Caucasian and 26\% were Hispanic. Twenty two per cent of the workers had not finished high school; $61 \%$ had completed high school or received a high school equivalency degree. Only $6 \%$ of the workers had any formal technical training. Their mean income was \$37 700 per year. The workers were not evenly distributed among industries. Thirty five per cent and 39\% of the workers were employed in boat building and truck and recreational vehicle manufacturing, respectively.

\section{Styrene monitoring results}

Air styrene concentrations ranged from $<1$ to $142 \mathrm{ppm}$, with a median of $9 \mathrm{ppm}$. Forty four $(10.6 \%)$ of the samples were below the limit of detection. Blood styrene concentrations ranged from $<0.001$ to $2.05 \mathrm{mg} / \mathrm{l}$ with a median of $0.0089 \mathrm{mg} / \mathrm{l}$. Twenty seven $(7.0 \%)$ of the blood styrene samples were below the limit of detection. Blood and air styrene concentrations were highly correlated with one another $(r=0.77, \mathrm{p}<0.001)$.

\section{Bivariate analyses}

Table 2 shows the results of bivariate analyses for variation in blood and air styrene levels and serum PRL concentrations with potential confounding variables. Female gender was associated with higher PRL and lower air and blood styrene levels. The negative effect of age on styrene levels appeared to be a function of the fact that younger workers were more likely to be employed in higher exposure job categories such as laminator, while older workers were more likely to be employed in lower exposure categories such as manager. Similarly, income was significantly negatively correlated with both measures of styrene exposure, likely due to the fact that

Table 1 Demographic characteristics of the study population

\begin{tabular}{ll}
\hline & No. (\%) \\
\hline Gender & \\
Male & $259(85.8)$ \\
Female & $43(14.2)$ \\
Race & $204(67.5)$ \\
Caucasian & $78(25.8)$ \\
Hispanic & $3(1.0)$ \\
African American & $12(4.0)$ \\
Asian & $4(1.3)$ \\
Native American & \\
Industry & $11(3.6)$ \\
Bath tub & $107(35.4)$ \\
Boat building & $44(14.6)$ \\
Pipe and tank manufacture & $117(38.7)$ \\
Truck and RV manufacture & $23(7.6)$ \\
Boat repair & $11(3.6)$ \\
Academic education & $55(18.2)$ \\
Less than grade school & $40(13.2)$ \\
Finished grade school & $146(48.3)$ \\
High school equivalency degree & $21(7.0)$ \\
Finished high school & $17(5.6)$ \\
At least one year college & $12(3.9)$ \\
Associates degree & $18(6.0)$ \\
Completed college & Mean (range) \\
Any technical training & $35.6(18.1-61.1)$ \\
\hline & $37.7(19.5-58.4)$ \\
\hline Age & $3.8(0-30.7)$ \\
Men & $6.3(0-39.0)$ \\
Women & $37.7(19.5-58)$ \\
Years in current job & $25.9(18.4-44.9)$ \\
Years as a laminator & \\
Body income in thousands of dollars & \\
\hline Some of the percentages may not add to 100 due to additional categories \\
with very few individuals. \\
*eight in kg divided by height in m². \\
$\quad$ \\
\hline
\end{tabular}


lower paid individuals were more likely to work in the higher styrene exposure job categories. Both age and income were also negatively correlated with serum PRL levels. The associations between job category and the styrene exposure measures were significant, with laminators having the highest air and blood styrene levels (data not shown). Therefore, job category and income were viewed as surrogates for exposure and were not included in the regression models. Similarly, air acetone concentrations were highly correlated with air and blood styrene concentrations and were therefore not included in the regression models. However, separate regression models in which acetone was the exposure variable were performed and revealed no significant association between air acetone concentration and serum PRL (data not shown). Ethnicity was significantly associated with both measures of styrene exposure, and tended to be associated with PRL levels. Non-Caucasians had higher blood and air styrene and higher PRL levels than Caucasians. These results suggest that ethnicity may also be a surrogate for exposure, rather than an independent predictor of PRL. Use of personal protective equipment in the form of respirators, gloves, and protective clothing was significantly associated with higher air and blood styrene concentrations. Both air and blood styrene concentrations varied significantly with time of day and season of the year, but serum PRL concentrations did not.

When smoking was categorised by number of cigarettes per day, the association between air styrene and cigarettes smoked per day approached statistical significance, but did not follow a monotonic dose-response. PRL decreased in a dose dependent, statistically significant manner with increasing number of cigarettes smoked per day. Consumption of alcoholic beverages was positively associated with both air and blood styrene concentrations. However, this was statistically significant only for air styrene, indicating differential rates of alcohol consumption among styrene exposure categories. The relation between PRL and alcohol consumption approached statistical significance, but did not follow a monotonic dose-response. The consumption of food or beverages from styrofoam containers was not significantly associated with blood styrene concentrations. Although the tables show prescription, non-prescription, and recreational drug use as dichotomised variables for the sake of brevity, the effects of different categories of drugs (for example, antidepressants or oral contraceptives) were evaluated for their effects on PRL and styrene. Prescription drug use was significantly associated with blood and air styrene. This appeared due to the lower styrene levels in women users of oral contraceptives or hormone replacement therapy. PRL levels did not vary with prescription drug use.

\section{Association between styrene exposure measures and serum prolactin}

The summary models are described in table 4, followed by more detailed models describing the acute exposure response in table 5. Table 4 shows the models including the potential confounding variables gender, smoking, and age. Increasing blood styrene levels were significantly associated with increasing serum PRL concentrations in the acute model. This model also had the highest coefficient for exposure, suggesting the strongest association between PRL and styrene. The anti-log of the styrene exposure coefficient was 2.06, indicating that serum PRL tends to increase 2.06-fold for every 10 -fold increase in the blood styrene concentration. The coefficient for subchronic blood styrene was lower than for acute blood styrene, and the relation between subchronic blood styrene and serum PRL did not attain statistical significance (anti-log of $\mathrm{B}=1.90, \mathrm{p}=0.105$ ). The coefficient estimates for air styrene as the exposure measure in the acute and subchronic models and for the chronic exposure indices did not differ appreciably from one another and were nearly an order of magnitude lower than the coefficient for acute blood styrene (table 4). Moreover, inclusion of subchronic and chronic indices in the same model with acute blood styrene or with acute air styrene did not appreciably improve the $\mathrm{R}^{2}$ of the models (data not shown). Women tended to have higher serum PRL levels than men, and the effect of gender was significant in the acute models (table 4). Smoking was statistically significantly associated with decreased serum PRL concentration in all of the models (table 4). Increasing age was also associated with decreasing PRL concentrations; however, this association was statistically significant only in the subchronic and chronic models (table 4).

The first two rows in table 5 show that serum PRL tended to increase with styrene exposure in both men and women and for both session 1 and session 2. For men, the coefficients for styrene were quite similar between the two sessions $(\mathrm{B}=0.300$ and $\mathrm{B}=0.377$ ), lending credence to the presence of a meaningful effect. The effect of exposure was not statistically significant for women, likely attributable to the smaller numbers of subjects. Excluding age from the models increased the strength of the associations between styrene exposure and PRL for men (table 5, rows 3 and 4).

To further address the issue of whether styrene acutely affects serum PRL concentrations and to further address the effects of age and gender, we also examined acute effects across sessions. If the effect of styrene on PRL is a pronounced acute effect, then one should be able to detect a change in PRL as a function of the change in acute exposure. This also eliminates age and individual differences in the analyses by using each subject as his or her own control. It may also increase the power to detect an effect of styrene exposure on PRL because it makes use of the repeated measurements of styrene and PRL during two study sessions. The results showed that the coefficient for change in styrene in blood was not statistically significant for men, whereas it was for women (table 5, rows 5 and 6). This shows a significant positive effect of styrene on PRL in women. Further, it supports the hypothesis that the effect of styrene exposure on PRL concentration is an acute effect, as opposed to a subchronic or chronic effect. An examination of the scatter plots revealed that the variation in PRL levels between session 1 and session 2 for men was not pronounced (Pearson correlation of 0.819 ). However, PRL levels varied considerably between sessions among women (Pearson correlation of 0.400 ). This may well explain the difference in the statistical significance of change in styrene as a predictor of change in PRL between men and women.

Logistic regression analysis with adjustment for gender, age, and smoking revealed that workers with time weighted average styrene exposures above $20 \mathrm{ppm}$ were more likely to have clinically increased serum PRL concentrations (greater than $15 \mathrm{ng} / \mathrm{ml}$ for men or $20 \mathrm{ng} / \mathrm{ml}$ for women; OR $=3.69$, $95 \%$ CI 1.39 to 9.79 ) than workers with exposures below $20 \mathrm{ppm}$.

\section{DISCUSSION}

The current study shows significant positive associations between blood styrene concentrations and serum PRL concentrations in men and women. Previous studies have shown a similar relation using the urinary styrene metabolites phenylglyoxylic acid and mandelic acid as biomarkers of styrene exposure in cross-sectional studies. ${ }^{11-13}{ }^{19}$ In the current study, multiple air and blood styrene measurements were obtained over time in the same individuals, permitting the modelling of the associations between acute, subchronic, and chronic styrene exposure indices on PRL secretion. The 
Table 2 Bivariate analyses of the effects of selected demographic, lifestyle, and occupational variables on blood and air styrene concentrations

\begin{tabular}{|c|c|c|c|c|c|}
\hline & & $\begin{array}{l}\text { Blood styrene, mean (SEM), } \\
\mathrm{mg} / \mathrm{l}\end{array}$ & p value* & $\begin{array}{l}\text { Air styrene, mean (SEM), } \\
\text { ppm }\end{array}$ & p value* \\
\hline \multirow[t]{2}{*}{ Gender } & Male & $0.27(0.02)$ & 0.029 & $20.5(1.6)$ & 0.031 \\
\hline & Female & $0.13(0.02)$ & & $11.5(2.0)$ & \\
\hline \multirow[t]{5}{*}{ Industry } & Bathtub & $0.21(0.08)$ & 0.019 & $19.4(7.5)$ & $<0.001$ \\
\hline & Boat build & $0.21(0.03)$ & & $14.0(1.9)$ & \\
\hline & Pipe, tank & $0.21(0.05)$ & & $16.5(2.0)$ & \\
\hline & Truck, RV & $0.33(0.04)$ & & $27.3(2.9)$ & \\
\hline & Boat repair & $0.09(0.02)$ & & $9.2(1.4)$ & \\
\hline \multirow[t]{2}{*}{ Respirator use } & No & $0.23(0.02)$ & 0.088 & $15.9(1.3)$ & 0.001 \\
\hline & Yes & $0.33(0.05)$ & & $32.6(4.6)$ & \\
\hline \multirow[t]{2}{*}{ Glove use } & No & $0.20(0.03)$ & 0.009 & $15.8(1.8)$ & 0.003 \\
\hline & Yes & $0.32(0.03)$ & & $24.5(2.4)$ & \\
\hline \multirow[t]{2}{*}{ Working in a confined space } & No & $0.22(0.03)$ & 0.152 & $16.1(1.5)$ & 0.002 \\
\hline & Yes & $0.29(0.04)$ & & $25.2(3.0)$ & \\
\hline \multirow[t]{2}{*}{ Recreational drug use } & No & $0.20(0.05)$ & 0.350 & $16.0(2.4)$ & 0.335 \\
\hline & Yes & $0.26(0.02)$ & & $19.8(1.6)$ & \\
\hline \multirow[t]{2}{*}{ Non-prescription drug use } & No & $0.23(0.03)$ & 0.453 & $18.7(2.4)$ & 0.745 \\
\hline & Yes & $0.26(0.03)$ & & $19.6(1.8)$ & \\
\hline \multirow[t]{2}{*}{ Prescription drug use } & No & $0.16(0.04)$ & 0.020 & $13.4(2.3)$ & 0.007 \\
\hline & Yes & $0.28(0.03)$ & & $21.1(1.7)$ & \\
\hline \multirow[t]{2}{*}{ Styrofoam food or drink containers } & No & $0.25(0.03)$ & 0.888 & $21.1(2.4)$ & 0.310 \\
\hline & Yes & $0.25(0.03)$ & & $18.1(1.8)$ & \\
\hline \multirow[t]{4}{*}{ Current no. of cigarettes per day } & $<1$ & $0.25(0.03)$ & 0.689 & 19.1 (1.9) & 0.131 \\
\hline & $1-10$ & $0.26(0.06)$ & & $24.2(4.6)$ & \\
\hline & $11-20$ & $0.21(0.04)$ & & $13.6(2.1)$ & \\
\hline & $>20$ & $0.32(0.12)$ & & $24.5(6.3)$ & \\
\hline \multirow{4}{*}{$\begin{array}{l}\text { Current no. of alcoholic beverages } \\
\text { per week }\end{array}$} & $<1$ & $0.21(0.03)$ & 0.272 & $14.4(1.6)$ & 0.003 \\
\hline & $1-7$ & $0.31(0.04)$ & & $23.9(3.0)$ & \\
\hline & $8-21$ & $0.25(0.06)$ & & $20.8(3.4)$ & \\
\hline & $>21$ & $0.23(0.11)$ & & $38.3(12.1)$ & \\
\hline \multirow[t]{3}{*}{ Ethnicity } & Caucasian & $0.20(0.02)$ & 0.007 & $15.2(1.4)$ & $<0.001$ \\
\hline & Hispanic & $0.34(0.05)$ & & $27.2(3.4)$ & \\
\hline & Othert & $0.37(0.11)$ & & $30.5(9.1)$ & \\
\hline \multirow[t]{4}{*}{ Season } & Dec-Feb & $0.38(0.05)$ & 0.016 & $33.8(4.2)$ & $<0.001$ \\
\hline & Mar-May & $0.20(0.07)$ & & $12.2(3.1)$ & \\
\hline & June-Aug & $0.24(0.03)$ & & $15.8(1.7)$ & \\
\hline & Sep-Nov & 0.19 (0.03) & & 14.4 (1.9) & \\
\hline \multirow[t]{3}{*}{ Time of day } & $21: 00-4: 59$ & $0.52(0.12)$ & 0.001 & $45.3(8.2)$ & $<0.001$ \\
\hline & $13: 00-16: 59$ & $0.23(0.02)$ & & $17.2(1.3)$ & \\
\hline & 17:00-20:59 & $0.15(0.04)$ & & $11.0(2.8)$ & \\
\hline \multirow{5}{*}{\multicolumn{2}{|c|}{$\begin{array}{l}\text { Age } \\
\text { Body mass index } \neq \\
\text { Income } \\
\text { Air acetone }\end{array}$}} & Correlation coefficient & & Correlation coefficient & \\
\hline & & -0.227 & $<0.001$ & -0.291 & $<0.001$ \\
\hline & & 0.054 & 0.379 & -0.007 & 0.911 \\
\hline & & -0.108 & 0.079 & -0.163 & 0.006 \\
\hline & & 0.250 & $<0.001$ & 0.349 & $<0.001$ \\
\hline
\end{tabular}

coefficients for styrene differed appreciably among the five models, in that the association between blood styrene concentration and serum PRL concentration in the acute model was stronger than for the other models. Moreover, for women the difference in blood styrene concentrations between session 2 and session 1 was a statistically significant predictor of both serum PRL concentration in session 2 and of the difference in serum PRL concentration between session 2 and session 1. Taken together, these findings provide evidence of an acute effect of styrene exposure on serum PRL concentration, as opposed to a subchronic or chronic effect. Moreover, additional analysis showed that individuals exposed to mean air styrene levels above the ACGIH TLV are at significantly greater risk of having clinically increased serum PRL concentrations than are those exposed to air styrene levels below $20 \mathrm{ppm}$.

The observation that the relation between blood styrene and PRL was a stronger relation than that between personal breathing zone air styrene and PRL has several possible explanations. First, although the primary route of styrene absorption is by inhalation, there is also limited absorption of styrene by the dermal route. ${ }^{121}$ Second, some of the subjects were using respirators while working with styrene, while others were not. In the bivariate analyses, we observed 1.5fold higher mean blood styrene levels in the workers who wore respirators than in those who did not; whereas the mean personal breathing zone styrene levels were 2.1 times higher in the workers who wore respirators compared to those who did not (table 2). This indicates that the respirators were protective, but did not completely prevent absorption of styrene. Third, rates of styrene absorption and excretion may vary among individuals, ${ }^{24}$ resulting in different blood styrene concentrations for the same air styrene exposure level. Finally, if the association between styrene exposure and PRL is an acute effect, one would expect a stronger association between blood styrene measured at the same time as PRL than with air styrene averaged over the work shift.

The finding in the present study that styrene exposure enhances PRL secretion is consistent with previous reports 


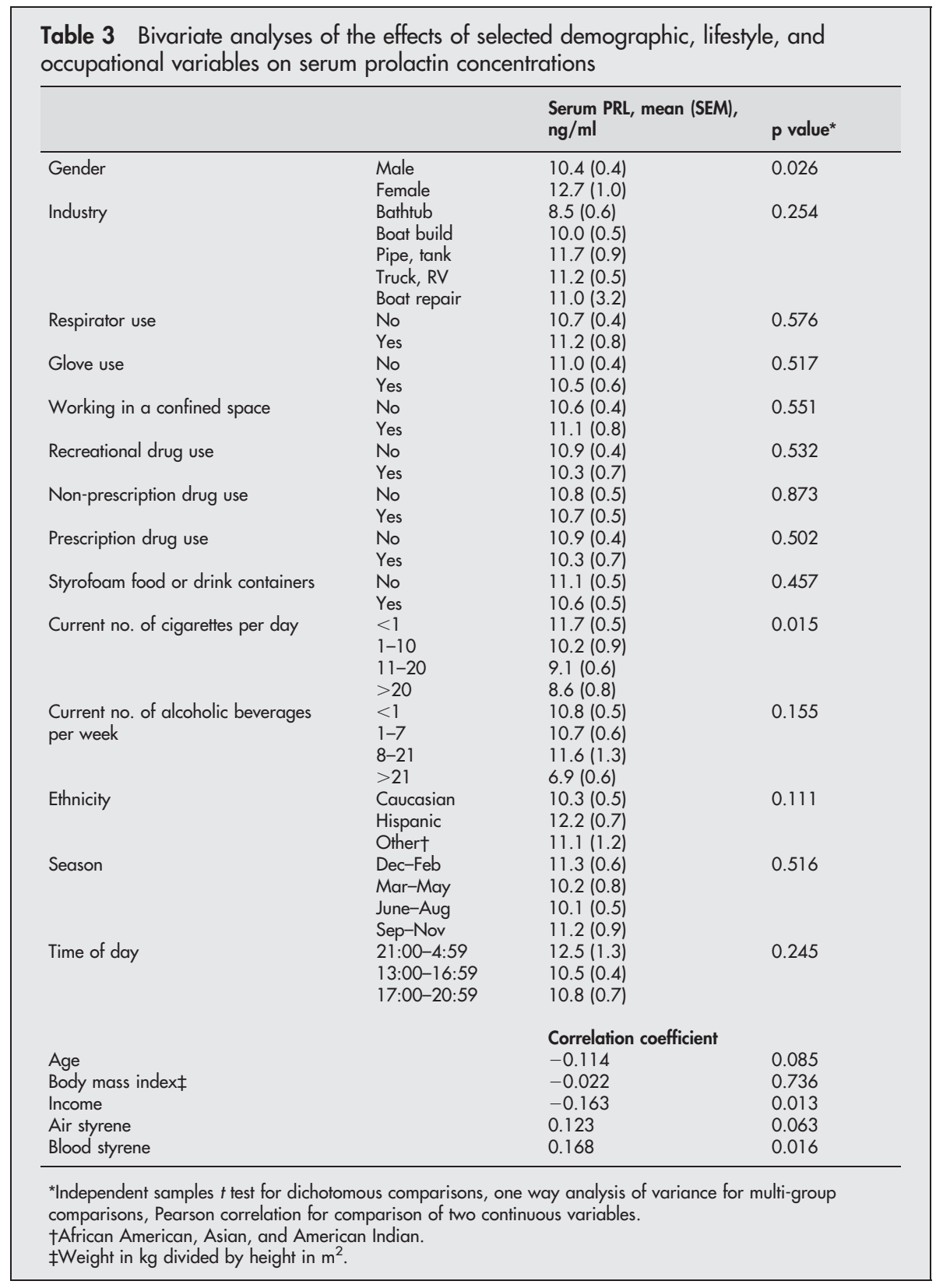

that styrene exposure depletes tuberoinfundibular dopamine concentrations in experimental animals, ${ }^{17}{ }^{18}$ which would result in increased pituitary PRL secretion. Depletion of dopamine occurs without changes in the rates of dopamine synthesis or degradation or in dopamine receptor number or affinity. ${ }^{17}{ }^{18}$ The styrene metabolites PGA and phenylglycine (the latter is formed by amination of PGA) mimic the effects of styrene on dopamine levels. ${ }^{25}$ Mutti and coworkers hypothesised that phenylglycine may compete with dopamine for reuptake into the nerve terminal, increasing the dopamine available for breakdown and decreasing tuberoinfundibular dopamine levels. ${ }^{17} 25$ This hypothesised mechanism would predict a rapid effect of styrene exposure on PRL secretion because no DNA transcription or protein synthesis is required and because styrene metabolism to PGA occurs rapidly. ${ }^{21}$

Severe hyperprolactinaemia, with PRL levels greater than $200 \mathrm{ng} / \mathrm{ml}$, occurs in patients with pituitary prolactinomas. Hyperprolactinaemia in these patients is associated with infertility, impotence, and galactorrhoea. ${ }^{26}$ Increases of serum
PRL above the upper limit of the range of normal, but below $100 \mathrm{ng} / \mathrm{ml}$, as was observed in some subjects in the present study, are not associated with these symptoms. However, there may be more subtle health effects associated with chronic low level increases in serum PRL. Recently, the immunomodulatory role of PRL has begun to be appreciated. PRL stimulates lymphocyte proliferation, antibody production, and macrophage and natural killer cell function in vitro. ${ }^{16}$ Increased PRL levels cause inflammation of the lateral prostate in male rats. ${ }^{27} 28$ It has been hypothesised that hyperprolactinaemia may also play a role in the development of non-bacterial prostatitis in humans ${ }^{28}$ However, the effects of low level increases of serum PRL levels on immune system function and development of prostatitis in humans have not yet been investigated.

Several factors other than styrene were noted to affect PRL levels in the current study. Serum PRL decreased in a dose dependent manner with number of cigarettes smoked per day. This negative effect of smoking on PRL concentrations is in agreement with previous studies. ${ }^{29}$ Serum PRL was also 
Table 4 Relation between styrene exposure variables and serum prolactin concentration adjusted for gender, age, and smoking using multiple linear regression

\begin{tabular}{|c|c|c|c|c|}
\hline Model & B & SE & $p$ value & $n$ \\
\hline \multicolumn{5}{|l|}{ Acute } \\
\hline Blood styrene & 0.314 & 0.137 & 0.023 & \multirow[t]{5}{*}{205} \\
\hline Gender & 0.0825 & 0.039 & 0.035 & \\
\hline Age & -0.0012 & 0.001 & 0.359 & \\
\hline Cigarettes per day & -0.0039 & 0.001 & 0.006 & \\
\hline Intercept & 1.090 & 0.063 & $<0.001$ & \\
\hline Air styrene & 0.0548 & 0.028 & 0.053 & \multirow[t]{5}{*}{227} \\
\hline Gender & 0.0820 & 0.038 & 0.030 & \\
\hline Age & -0.0011 & 0.001 & 0.424 & \\
\hline Cigarettes per day & -0.0042 & 0.001 & 0.002 & \\
\hline Intercept & 1.061 & 0.073 & $<0.001$ & \\
\hline \multicolumn{5}{|l|}{ Subchronic } \\
\hline Blood styrene & 0.279 & 0.171 & 0.105 & \multirow[t]{5}{*}{175} \\
\hline Gender & 0.0179 & 0.042 & 0.669 & \\
\hline Age & -0.0031 & 0.001 & 0.034 & \\
\hline Cigareftes per day & -0.0071 & 0.002 & $<0.001$ & \\
\hline Constant & 1.088 & 0.061 & $<0.001$ & \\
\hline Air styrene & 0.0563 & 0.031 & 0.074 & 175 \\
\hline Gender & 0.0093 & 0.041 & 0.823 & \\
\hline Age & -0.0030 & 0.002 & 0.051 & \\
\hline Cigareftes per day & -0.0063 & 0.002 & $<0.001$ & \\
\hline Intercept & 1.045 & 0.077 & $<0.001$ & \\
\hline \multicolumn{5}{|l|}{ Chronic } \\
\hline $\begin{array}{l}\text { Total years in industry * total average air } \\
\text { styrene }\end{array}$ & 0.0403 & 0.021 & 0.060 & \multirow[t]{5}{*}{175} \\
\hline Gender & 0.0128 & 0.042 & 0.758 & \\
\hline Age & -0.0040 & 0.001 & 0.005 & \\
\hline Cigareftes per day & -0.0066 & 0.002 & $<0.001$ & \\
\hline Intercept & 1.081 & 0.063 & $<0.001$ & \\
\hline $\begin{array}{l}\text { Total years in facility * total average air } \\
\text { styrene }\end{array}$ & 0.0263 & 0.023 & 0.255 & \multirow[t]{5}{*}{175} \\
\hline Gender & 0.0120 & 0.042 & 0.777 & \\
\hline Age & -0.0038 & 0.001 & 0.007 & \\
\hline Cigarettes per day & -0.0064 & 0.002 & $<0.001$ & \\
\hline Intercept & 1.104 & 0.064 & $<0.001$ & \\
\hline
\end{tabular}

The log (base 10) transformation was used for the concentrations of styrene in air (ppm), styrene in blood (mg/l), and serum prolactin (PRL, $\mathrm{ng} / \mathrm{mll}$ ). For the acute exposure model air or blood styrene concentrations obtained on the same day as the PRL concentration were used. The outcome variable for the acute models was the PRL concentration obtained on the same day as the blood and air styrene concentrations. For the subchronic exposure models, air or blood styrene values were averaged over all the study sessions (2 or 3 sessions, approximately one year apart) for each subject who participated in more than one session. For the chronic exposure models all the air styrene values for the individual were averaged and multiplied times the number of years the subject had worked in that industry or facility. For the subchronic and chronic models the outcome variable was the serum PRL

concentration obtained during the last session for that individual (the second or third session). Gender was coded as 0 for men and 1 for women.

$\mathrm{n}$, number of observations included in analysis. For the acute models only the data from session 1 were used. For subchronic and chronic models, only subjects for whom there was data from more than one study session were included.

Table 5 Relation between acute blood styrene and serum prolactin concentrations; effects of gender, study session (S1 and S2), and age

\begin{tabular}{|c|c|c|c|c|c|c|}
\hline \multirow{2}{*}{$\begin{array}{l}\text { Linear regression model (exposure variable, } \\
\text { outcome variable) }\end{array}$} & \multicolumn{3}{|l|}{ Men } & \multicolumn{3}{|c|}{ Women } \\
\hline & B & p value & $\mathbf{n}$ & B & $p$ value & $\mathbf{n}$ \\
\hline S1 acute blood styrene, S1 PRL* & 0.300 & 0.019 & 172 & 0.226 & 0.822 & 33 \\
\hline S2 acute blood styrene, S2 PRL* & 0.377 & 0.044 & 145 & 0.726 & 0.459 & 23 \\
\hline S1 acute blood styrene, S1 PRL $†$ & 0.357 & 0.004 & 172 & 0.058 & 0.953 & 33 \\
\hline S2 acute blood styrene, S2 PRL† & 0.473 & 0.011 & 145 & 0.649 & 0.458 & 23 \\
\hline S2 minus S1 blood styrene, S2 PRL & 0.69 & 0.434 & 106 & 16.98 & 0.043 & 20 \\
\hline S2 minus S1 blood styrene, S2 minus S1 PRL§ & 1.05 & 0.272 & 106 & 22.69 & 0.022 & 20 \\
\hline
\end{tabular}

*The log base 10 transformations of PRL and styrene were used. Models also included age and number of cigarettes smoked per day.

†The log base 10 transformations of PRL and styrene were used. Models also included number of cigarettes smoked per day.

†Models also included S1 PRL, difference in number of cigarettes smoked per day, elapsed years, and difference in time of day blood was drawn between session

1 and 2 samples.

§Models also included difference in number of cigarettes smoked per day, elapsed years, and difference in time of day blood was drawn between session 1 and 2 samples. 
negatively correlated with age. PRL levels in women have been reported to decline after menopause in some, but not all, studies. ${ }^{30} 31$ In men mean daytime PRL levels have been reported to not vary with age ${ }^{31}$ or to increase with age. ${ }^{30} \mathrm{~A}$ more detailed study in which multiple serum samples were obtained over many hours found that aging in men is associated with a decline in the nocturnal rise in PRL levels, with daytime levels unaffected. ${ }^{32}$ In light of the inconsistent effects of age on PRL reported in the literature, one must also consider the possibility that the apparent effect of age on PRL concentrations in the bivariate analyses in the current study may have been due to the relation between age and exposure, as age was also inversely correlated with styrene exposure.

There are several limitations to the current study. First, one cannot exclude with certainty that the apparent effect of styrene on PRL secretion was in fact due to another exposure that was highly correlated with styrene. The most obvious of these, acetone, was measured as part of the study. Separate analyses that used acetone as the exposure variable rather than styrene found no significant association with serum PRL levels (data not shown). The next two limitations relate to our ability to clearly distinguish between acute, subchronic, and chronic effects of styrene on PRL. Although acute blood styrene appeared to be a stronger predictor of serum PRL concentration than the subchronic or chronic exposure indices, the acute, subchronic, and chronic styrene exposure measures were highly correlated with one another $(\mathrm{p}<0.001$, Pearson correlation coefficient for acute with subchronic and subchronic with chronic air styrene concentrations). Thus, one cannot unequivocally separate acute, subchronic, and chronic effects based on the models in table 4 alone. Second, since the acute effects were based on measures of exposure obtained for each subject on each date of testing, there should have been relatively little measurement error (in exposure) in the coefficients from regression analyses of acute effects. However, since the number of repeated measurements per subject was relatively small, measurement error effects could have diminished the corresponding coefficients in regression analyses for subchronic and chronic effects. Thus, we cannot rule out the possibility that longer term exposures to styrene also gave rise to subchronic or chronic effects on serum PRL. Both of these limitations seem less likely in light of the statistically significant association between the change in blood styrene between sessions 1 and 2 and the change in serum PRL between sessions 1 and 2 in women, which supports an acute effect. A final limitation is that the female subjects were not asked whether they were breast feeding, which is a physiological state associated with increased serum PRL levels. However, there is no reason to believe that women with high PRL levels due to lactation would have been differentially distributed to high styrene exposure.

In summary, the results of the present study confirm that styrene exposure increases serum PRL levels in a dose dependent manner in both men and women. The summary model predicts that serum PRL levels increase 2.06-fold for every 10 -fold increase in blood styrene levels. Blood styrene concentration measured at the same time as serum PRL concentration was more strongly associated with serum PRL than were personal breathing zone styrene concentration or calculated subchronic and chronic styrene exposure indices. For women, the change in blood styrene between sessions 1 and 2 was a significant predictor of the change in serum PRL between sessions 1 and 2 . These results support an acute effect of styrene on PRL concentration. Individuals exposed to time weighted average air styrene concentrations greater than 20 ppm were significantly more likely to have serum PRL levels greater than the upper limit of the normal reference ranges. These data provide additional evidence for biological effects of styrene at levels of exposure currently permissible in workplaces in many countries.

\section{ACKNOWLEDGEMENTS}

The authors thank the employers for providing access to their workplaces and the study subjects for participating in the study. We also acknowledge the contribution from the entire styrene data collection field team without whom this analysis would not have been possible. We thank Dr Anita Iannucci of the University of California Irvine Center for Statistical Consulting and Dr William Griffith of the University of Washington Institute for Risk Analysis and Risk Communication for their statistical expertise.

Financial support for this study was provided by NIDR R01-DE11712; NCI R01-CA6943; NIEHS Superfund Program ES04696 and ES07033; NIEHS training grant T32ES07018; the Center for Occupational and Environmental Health at the University of California at Irvine; the NIEHS Center for Ecogenetics and Environmental Health at the University of Washington (P30 ES07033-03).

\section{Authors' affiliations}

U Luderer, T Shay, Division of Occupational and Environmental Medicine, Department of Medicine, University of California, Irvine, CA, USA

R Tornero-Velez, National Exposure Research Laboratory,

Environmental Protection Agency, Research Triangle Park, NC, USA

S Rappaport, Department of Environmental Sciences and Engineering, School of Public Health, University of North Carolina, Chapel Hill, NC, USA

N Heyer, D Echeverria, Battelle Center for Public Health Research and Evaluation (CPHRE), Seattle, WA, USA

\section{REFERENCES}

1 NIOSH. Criteria for a recommended standard: occupational exposure to styrene. Cincinnati, OH: US Department of Health and Human Services, National Institute of Occupational Safety and Health, Robert A. Taft Laboratories, 1983:250.

2 Cherry N, Waldron HA, Wells GG, et al. An investigation of the acute behavioral effects of styrene on factory workers. $\mathrm{Br} J$ Ind Med 1980;37:234-40

3 Triebig G, Lehrl S, Weltle D, et al. Clinical and neurobehavioral study of the acute and chronic neurotoxicity of styrene. $\mathrm{Br} J$ Ind Med 1989:46:799-804

4 Cherry N, Rodgers B, Venables $\mathrm{H}$, et al. Acute behavioral effects of styrene exposure: a further analysis. Br J Ind Med 1981;38:346-50.

5 Mutti A, Mazzucchi A, Rustichelli P, et al. Exposure-effect and exposureresponse relationships between occupational exposure to styrene and neuropsychological functions. Am J Ind Med 1984;5:275-86.

6 Letz R, Mahoney FC, Hershman DL, et al. Neurobehavioral effects of acute styrene exposure in fiberglass boatbuilders. Neurotoxicol Teratol 1990;12:665-8.

7 Yokoyama K, Araki S, Murata K. Effects of low level styrene exposure on psychological performance in FRP boat laminating workers. Neurotoxicology 1992;13:551-6.

8 Gobba F, Galassi C, Imbriani M, et al. Acquired dyschromatopsia among styrene-exposed workers. J Occup Med 1991;33:761-5.

9 Campagna D, Mergler D, Huel G, et al. Visual dysfunction among styreneexposed workers. Scand J Work Environ Health 1995;21:382-90.

10 Triebig G, Stark T, Ihrig A, et al. Intervention study on acquired color vision deficiencies in styrene-exposed workers. J Occup Environ Med 2001;43:494-500.

11 Arfini G, Mutti A, Vescovi P. Impaired dopaminergic modulation of pituitary secretion in workers occupationally exposed to styrene: further evidence from PRL response to TRH stimulation. J Occup Med 1987:129:826-30.

12 Bergamaschi E, Mutti A, Cavazzini S, et al. Peripheral markers of neurochemical effects among styrene-exposed workers. Neurotoxicology 1996:17:753-60.

13 Bergamaschi E, Smargiassi A, Mutti A, et al. Peripheral markers of catecholaminergic dysfunction and symptoms of neurotoxicity among styreneexposed workers. Int Arch Occup Environ Health 1997;69:209-14.

14 ACGIH. Threshold limit values for chemical substances and physical agents. Biological exposure indices. Cincinnati, $\mathrm{OH}$ : American Conference of Government Industrial Hygienists, 2001.

15 Anon. Information on the regulatory treatment of styrene. International Styrene Industry Forum. http://www.styreneforum.org/regulatory_index.htm, 2001.

16 Ben-Jonathan N, Mershon JL, Allen DL, et al. Extrapituitary prolactin: distribution, regulation, functions, and clinical aspects. Endocr Rev 1996; 17:639-67.

17 Mutti A, Falzoi M, Romanelli A, et al. Regional alterations of brain catecholamines by styrene exposure in rabbits. Arch Toxicol 1984;55:173-7. 
18 Chakrabarti SK. Altered regulation of dopaminergic activity and impairment in motor function in rats after subchronic exposure to styrene. Pharmacol Biochem Behav 2000;66:523-32.

19 Mutti A, Vescovi PP, Falzoi M, et al. Neuroendocrine effects of styrene on occupationally exposed workers. Scand J Work Environ Health 1984; 10:225-8.

20 Apostoli P, Alessandro G, Placidi D, et al. Metabolic interference in subjects occupationally exposed to binary styrene-acetone mixtures. Int Arch Occup Environ Health 1998;71:445-52.

21 Guillemin MP, Berode M. Biological monitoring of styrene: a review. Am Ind Hyg Assoc J 1988;49:497-505.

22 Tornero-Velez R, Waidyanatha S, Perez HL, et al. Determination of styrene and styrene-7,8-oxide in human blood by gas chromatography-mass spectrometry. J Chromatogr B 2001;757:59-68.

23 Tornero-Velez R, Waidyanatha S, Echeverria D, et al. Measurement of styrene-7,8-oxide and other oxidation products of styrene in air. J Environ Monit 2000;2:497-505.

24 Jang J-Y, Droz PO, Berode M. Ethnic differences in biological monitoring of several organic solvents. 1. Human exposure experiment. Int Arch Occup Environ Health 1997;69:343-9.
25 Mutti A Romanelli A, Falzoi $M$ et al. Styrene metabolism and striatal dopamine depletion in rabbits. Arch Toxicol 1985;(suppl 8):447-50. 26 Shimon I, Melmed S. Management of pituitary tumors. Ann Intern Med 1998;129:472-83.

27 Tangbanluekal L, Robinette CL. Prolactin mediates estradiol-induced inflammation in the lateral prostate of Wistar rats. Endocrinology 1993;132:2407-16.

28 Stoker TE, Robinette CL, Cooper RL. Maternal exposure to atrazine during lactation suppresses suckling-induced prolactin release and results in prostatitis in the adult offspring. Toxicol Sci 1999;52:68-79.

29 Fuxe K, Andersson K, Eneroth $\mathrm{P}$, et al. Neuroendocrine actions of nicotine and of exposure to cigarette smoke: medical implications. Psychoneuroendocrinology 1989;14:19-41.

30 Blackman MR. Pituitary hormones and aging. Endocrinol Metab Clin 1987; 16:981-94.

31 Maddox PR, Jones DL, Mansel RE. Basal prolactin and total lactogenic hormone levels by microbioassay and immunoassay in normal human sera. Acta Endocrinol (Copenh) 1991;125:621-7.

32 van Coevorden A, Mockel J, Laurent E, et al. Neuroendocrine rhythms and sleep in aging men. Am J Physiol 1991;260:E651-61. 